\title{
Mathematische Modelle in der Immuntherapie von Tumoren
}

\author{
Anna Kraut
}

\begin{abstract}
Biomedizinische Fragestellungen sind in den letzten Jahren zu einer populären Anwendung mathematischer Modellierung geworden. Im Gegensatz zu Wirtschafts- oder Klimasystemen lassen sich viele biologische Systeme im Labor leicht kontrollieren und manipulieren. Besonders geeignet sind in diesem Zusammenhang populationsdynamische Modelle für Evolution, die in der Arbeitsgruppe von Anton Bovier am Institut für Angewandte Mathematik in Bonn erforscht werden. Um diese effizient zu simulieren, werden stochastisch-deterministische Hybridalgorithmen entwickelt. Das Zusammenspiel zwischen mathematischer und medizinischer Forschung führt zu interessanten Fragestellungen für beide Seiten.
\end{abstract}

Inspiration aus der Biomedizin

Die Entwicklung von Tumoren ist eine besonders interessante Anwendung für mathematische Evolutionsmodelle. Durch die hohen Mutationsraten können evolutionäre Vorgänge innerhalb kürzester Zeit beobachtet und beeinflusst werden. Anders als zum Beispiel in der Evolution von Säugetieren können so Modell und Wirklichkeit direkt miteinander verglichen und aufeinander abgestimmt werden.

In Kooperation mit der Arbeitsgruppe von Michael Hölzel am Universitätsklinikum Bonn untersuchen wir die Immuntherapie von malignen Melanomen, einer schädlichen Form von Hautkrebs. Die Experimente in einem Mausmodell bilden die Basis des mathematischen Modells. Dabei passiert folgendes: Die Tumorzellen kommen in unterschiedlichen Formen vor. Normalerweise können sie durch ein für sie typisches Protein, ein sogenanntes Antigen, auf der Zelloberfläche identifiziert werden. Im Laufe der Therapie werden spezifische Immunzellen injiziert, die die Tumorzellen an diesem Antigen erkennen und töten. Zur selben Zeit werden von den aktiven Immunzellen allerdings auch Botenstoffe ausgeschüttet, unter deren Einfluss die Tumorzellen ihre Form wandeln und die Antigene nicht mehr zeigen. Dadurch sind sie nicht mehr angreifbar und die Therapie schlägt fehl.

Ziel der gemeinsamen Forschung ist es, die Vorgänge im Tumor besser zu verstehen, verschiedene Therapieansätze wie beispielsweise eine Kombination mit Chemotherapie durchzuspielen und Überlebensstrategien des Tumors zu untersuchen.

\section{Herleitung des Modells}

Unser mathematisches Modell ist ein Markovprozess, also ein stochastischer Prozess, dessen zukünftige Entwicklung einzig vom aktuellen Zustand und nicht von der Vergangenheit abhängt. Wir betrachten einen sogenannten Individuenbasierten Markovprozess, bei dem einzelne Akteure miteinander interagieren.
Der Zustand des Prozesses wird durch einen Populationsvektor beschrieben, der die Anzahl der Individuen mit verschiedenen Merkmalen angibt. Für Merkmale $x \in \mathcal{X}$ und $t \geq 0$ ist $n_{x}(t)$ die Anzahl der Individuen mit Merkmal $x$ zum Zeitpunkt $t$ und $n(t) \in \mathbb{N}^{\mathcal{X}}$.

Die Dynamik des Prozesses wird dadurch bestimmt, dass verschiedene Ereignisse zu zufälligen, exponentialverteilten Zeiten auftreten und den Zustand der Population verändern. Beispielsweise kann jedes Individuum mit Merkmal $x$ mit der Rate $b_{x}$ einen Nachkommen desselben Typs produzieren. Der Zustand wird dabei von $n(t) \mathrm{zu} n(t)+\delta_{x}$ verändert, wobei mit $\delta_{x}$ die entsprechenden Einheitsvektoren bezeichnet sind. Da $n_{x}(t)$ Individuen vom Typ $x$ existieren, passieren im Durchschnitt $b_{x} n_{x}(t)$ "Geburten“ pro Zeiteinheit, allerdings nicht regelmäßig, sondern in variierenden, zufälligen Abständen.

Für eine allgemeine Menge $\mathcal{E}$ von möglichen Ereignissen bezeichnen wir mit $v_{e}$ die entsprechende Zustandsveränderung und mit $r_{e}(n)$ die Gesamtrate, mit der diese auftritt. Im obigen Beispiel ( $e=$ Geburt vom Typ $x$ ) wäre $v_{e}=\delta_{x}$, und $r_{e}(n)=b_{x} n_{x}$ setzt sich aus der individuellen Rate und der Anzahl von Individuen zusammen. Ein Markovprozess wird durch seinen sogenannten Generator charakterisiert. In diesem Fall schreibt sich der Generator als

$$
\mathcal{L} \varphi(n)=\sum_{e \in \mathcal{E}}\left(\varphi\left(n+v_{e}\right)-\varphi(n)\right) r_{e}(n),
$$

für messbare, beschränkte $\varphi: \mathbb{N}^{\mathcal{X}} \rightarrow \mathbb{R}$.

Verschiedene Versionen dieses Prozesses wurden in den letzten Jahren auf theoretischer Ebene als Modell für Evolution untersucht. Dabei wurden vor allem die Mechanismen von Geburt, Tod, Mutation und Konkurrenz zwischen Individuen berücksichtigt. Von großem Interesse ist es dabei, den Prozess durch einfachere Prozesse anzunähern, um Aussagen über das Langzeitverhalten machen zu können.

Das Modell, das wir zur Untersuchung von Immuntherapie von Melanomen verwenden, ist von derselben Art. Es wird zwischen verschiedenen Typen von Individuen unterschieden, in diesem Fall zwischen normalen ( $\mathrm{Tum}^{1}$ ) und „versteckten“ (Tum² ${ }^{2}$ Tumorzellen, sowie Immunzellen und 


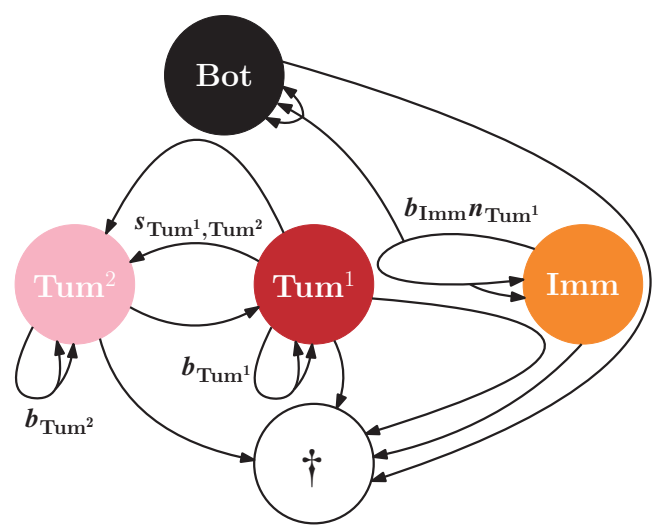

Interaktionsschema des Tumors. Jeder Pfeil steht für ein mögliches Ereignis, die Formeln beschreiben beispielhaft einige der individuellen Raten, mit denen die jeweiligen Ereignisse passieren.

Botenstoffen. Zusätzlich zu Geburt/Zellteilung, Tod (natürlich oder durch Konkurrenz) und Mutation müssen aber noch eine Reihe anderer Mechanismen miteinbezogen werden. Beispielsweise vermehren sich die Immunzellen nur in Anwesenheit der normalen Tumorzellen. Dadurch bekommt ihre (Gesamt-)Geburtenrate die Form von $b_{\text {Imm }} n_{\text {Imm }} n_{\text {Tum }}{ }$.

Des Weiteren können mehrere Ereignisse gekoppelt werden. Bei der Vermehrung der Immunzellen werden zum Beispiel $m$ Botenstoffe ausgeschüttet, was eine Zustandsänderung von $n \mathrm{zu} n+\delta_{\text {Imm }}+m \delta_{\text {Bot }}$ bedeutet, und wenn eine normale Tumorzelle ihre Form ändert, entspricht dies $v_{e}=-\delta_{\mathrm{Tum}^{1}}+\delta_{\mathrm{Tum}^{2}}$ und $r_{e}(n)=s_{\mathrm{Tum}^{1}, \mathrm{Tum}^{2}} n_{\mathrm{Tum}^{1}}$.

Die Hypothese darüber, wie die einzelnen Zellen und Moleküle im Tumor interagieren, wird in dem oben wiedergegebenen Schema dargestellt.

Ein solches Modell lässt sich immer dann anwenden, wenn Akteure mit exponentiellen Raten miteinander interagieren.

Nachdem das Experiment abstrahiert und die (vermeintlich) wichtigsten Mitspieler und Ereignisse identifiziert wurden, müssen die konkreten Parameter des Systems, beispielsweise $b_{\mathrm{Tum}^{1}}$ und $b_{\mathrm{Tum}^{2}}$, bestimmt werden. Dazu werden die experimentellen Daten ausgewertet und medizinisches Vorwissen genutzt.

Nun kann der stochastische Prozess algorithmisch implementiert werden, um verschiedene Szenarien zu simulieren.

\section{Stochastisch vs. deterministisch}

Der oben beschriebene Markovprozess erscheint realistischer als ein deterministisches Modell. Zellen teilen sich beispielsweise nicht genau alle paar Stunden, sondern zufällig mit einer gewissen durchschnittlichen Rate. Für die theoretische Betrachtung ist das kein Problem, allerdings kann die Simulation des stochastischen Modells sehr aufwendig sein.

Wir nutzen einen sogenannten Gillespie-Algorithmus. Er ist so aufgebaut, dass in jedem Schritt ein einzelnes Ereignis, wie beispielsweise eine Geburt, simuliert wird. Dafür wird zunächst die zufällige Wartezeit bis zum nächsten Ereignis simuliert und dann entschieden, welche der oben genannten Zustandsänderungen eintritt. Das Interessante ist, dass dabei nicht für jedes mögliche Ereignis eine eigene exponentielle Wartezeit generiert und dann die kleinste ausgewählt werden muss, um das nächste Ereignis zu bestimmen. Stattdessen werden die Eigenschaften exponentialverteilter Zufallsvariablen ausgenutzt. Das Minimum einer endlichen Menge unabhängiger, exponentialverteilter Zufallsvariablen ist wieder exponentialverteilt, mit der Summe der einzelnen Raten als Parameter. So muss nur eine einzige Zufallszeit generiert werden. Die Wahrscheinlichkeit, dass ein bestimmtes Ereignis dieses Minimum annimmt, ist dann proportional zu den einzelnen Raten und kann durch eine weitere Zufallszahl bestimmt werden.

In einer großen Population passieren jedoch sehr viele dieser Ereignisse in kurzer Zeit, da sich viele Zellen teilen, sterben etc. Als Konsequenz müssen sehr viele Schritte im Algorithmus gemacht werden, um eine interessante Zeitspanne zu simulieren.

Der Ausweg ist ein Resultat aus der theoretischen Forschung zu Individuen-basierten Markovprozessen. In den Achtzigerjahren zeigten Ethier und Kurtz, dass der reskalierte stochastische Prozess im Grenzwert großer Populationen gegen die Lösung eines Systems gewöhnlicher Differenzialgleichungen konvergiert. Dieses ist mit obiger Notation gegeben durch

$$
\frac{d}{d t} n_{x}(t)=\sum_{e \in \mathcal{E}}\left(v_{e}\right)_{x} r_{e}(n(t)) .
$$

In einer Art Gesetz der großen Zahlen mitteln sich dabei die stochastischen Fluktuationen um den Mittelwert, der durch die Differenzialgleichungen beschrieben ist, heraus. Dieses deterministische System lässt sich also nutzen, um in gewissen Fällen das stochastische System auch für endliche Populationen anzunähern und ist dabei mit Hilfe eines klassischen Runge-Kutta-Verfahrens sehr viel schneller zu simulieren.

Die Lösung ist allerdings nicht, von nun an ausschließlich das deterministische System zu betrachten. Gewisse Effekte werden dadurch nicht oder falsch abgebildet. Betrachtet man beispielsweise eine kleine Population, wie einen Tumor zu Beginn seines Wachstums, ist es sehr wichtig, in welcher Reihenfolge die verschiedenen Ereignisse eintreten. Auch wenn die Teilungsrate der Zellen größer ist als ihre Todesrate und die Gesamtwachstumsrate im deterministischen System somit positiv wäre, kann es passieren, dass die Zellen manchmal zufällig absterben, bevor sie sich teilen konnten, und der Tumor somit ausstirbt. Dieser Effekt kann im deterministischen System nie beobachtet werden. Dies hängt damit zusammen, dass auch eine exponentiell abfallende Funktion niemals Null erreicht. Besonders wichtig wird dies, wenn zum Beispiel nur noch wenige Tumor- und Immunzellen übrig sind. In diesem Fall ist es entscheidend, welcher Zelltyp zuerst ausstirbt. Davon hängt ab, ob der Tumor besiegt wird oder aber erneut wächst, wenn er nicht mehr bekämpft werden kann.

In unseren Simulationen kombinieren wir deshalb beide Verfahren. Gemeinsam mit der Arbeitsgruppe von Mar- 

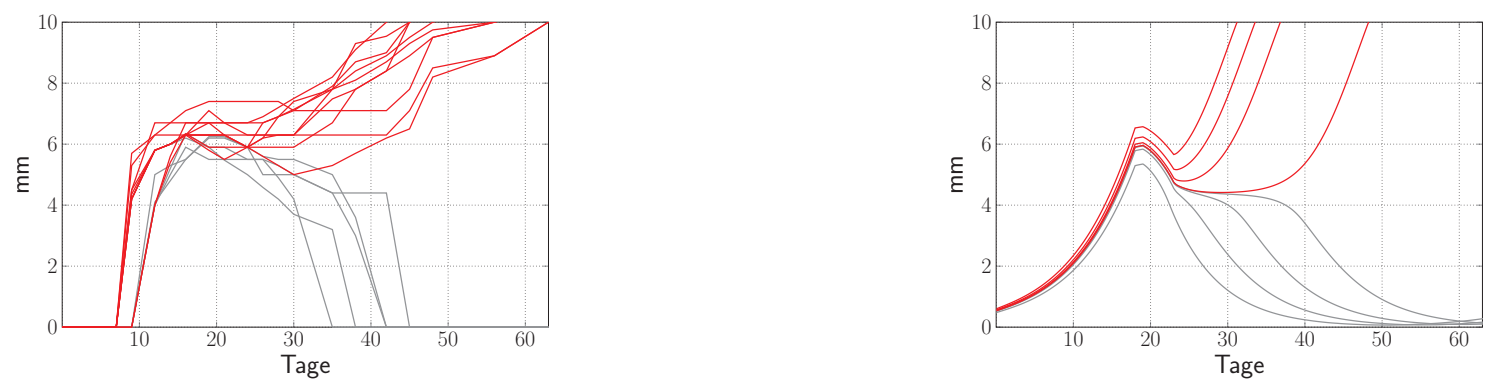

Tumordurchmesser im Zeitverlauf für verschiedene Mäuse im Experiment (links) und variierende Anfangsdaten in der Simulation (rechts). Zu Beginn wächst der Tumor unbehandelt, an Tag 18 wird die Therapie begonnen. Rote Kurven markieren die Fälle, in denen der Tumor der Therapie entwächst, während graue Kurven erfolgreich bekämpfte Tumore anzeigen.

tin Rumpf am Institut für Numerische Simulation haben wir einen Hybridalgorithmus entwickelt, in dem parallel häufige Ereignisse mit Hilfe des deterministischen Systems angenähert und seltene Ereignisse wie im stochastischen System simuliert werden. Dadurch wird Rechenzeit gespart und gleichzeitig bleiben die zufälligen Effekte in kleinen Populationen erhalten.

Die Frage ist nun, in welchen Situationen stochastische Effekte wichtig sind. Dies ist nicht nur in kleinen Populationen oder bei seltenen Ereignissen der Fall, sondern allgemein an "kritischen Punkten“ des deterministischen Systems. Damit gemeint sind Zustände des Prozesses, in denen kleine Schwankungen einen großen Effekt auf die weitere Entwicklung der Population haben. Dies kann auch im Inneren des Zustandsraums gegeben sein, wenn beispielsweise eine Bifurkation auftritt, also eine stetige Veränderung eines Parameters eine plötzliche qualitative Änderung des Verhaltens der Lösung verursacht.

Unsere aktuelle Forschung beschäftigt sich damit, wie solche kritischen Punkte am besten zu quantifizieren sind und wie ein Algorithmus diese während der Simulation effektiv detektieren kann, um adaptiv zwischen dem stochastischem und dem deterministischen Verfahren zu wechseln. Dabei soll natürlich die Rechenzeit nicht stark erhöht werden, um weiterhin einen Vorteil gegenüber der rein stochastischen Simulation zu wahren.

\section{Zurück zur Anwendung}

Ein kritischer Punkt im Inneren des Zustandsraums lässt sich auch im Beispiel der Immuntherapie von Melanomen beobachten. In der Abbildung oben ist zu sehen, wie die Ergebnisse der Simulationen die experimentellen Daten widerspiegeln. In beiden Fällen ist zu erkennen, dass die Therapie nur bei kleineren Tumoren erfolgreich ist. Ab ei- ner gewissen Schwellengröße kommt das Immunsystem nicht mehr hinterher und der Tumor kann der Therapie entwachsen. Diese Schwelle markiert eine Bifurkation des deterministischen Systems und im stochastischen System können mit denselben Anfangswerten beide Fälle erreicht werden.

Was aber ist der Mehrwert davon, mit einer Simulation die bereits experimentell ermittelten Daten reproduzieren zu können? Es ist zwar kein Beweis, aber doch ein starkes Indiz dafür, dass die zugrundeliegende Hypothese über die Mechanismen des biologischen Systems richtig war. Oftmals lassen sich die experimentellen Daten durch das erste mathematische Modell - unabhängig von der Parameterwahl nicht abbilden. Dann muss die Hypothese, und mit ihr das Modell, angepasst und möglicherweise erweitert werden. Dadurch lassen sich wichtige Mechanismen identifizieren und neue Forschungsfragen, auch für die Medizin, entwickeln. Darüber hinaus können Simulationen jenseits der Experimente gemacht werden, um diese zu validieren (natürliches Auftreten einer Mutante in der Simulation anstatt einer künstlich herbeigeführten Mutation im Experiment) und Vorhersagen zu treffen. Letztere sind, abhängig von den zugrundeliegenden experimentellen Daten und der daraus resultierenden Genauigkeit der Parameterbestimmung, quantitativ nicht verlässlich. Treten aber gewisse Effekte unabhängig von der Parameterwahl auf, lassen sich durchaus qualitative Vorhersagen treffen.

\section{Literatur}

[1] M. Hölzel, A. Bovier, and T. Tüting, Plasticity of tumor and immune cells: A source of heterogeneity and a cause for therapy resistance? Nature Reviews Cancer 13, 365-376 (2013).

[2] M. Baar, L. Coquille, H. Mayer, M. Hölzel, M. Rogava, T. Tüting, and A. Bovier. A stochastic model for immunotherapy of cancer. Scientific Reports 6, 24169 (2016).

Anna Kraut, Rheinische Friedrich-Wilhelms-Universität Bonn, Institut für Angewandte Mathematik, Hausdorff Center for Mathematics, Endenicher Allee 6o, 53115 Bonn kraut@iam.uni-bonn.de 